\section{Practice Plan customer service}

Practice Plan has always placed high quality service at the core of its business ethos. Now Practice Plan has announced the opening of its new dedicated Customer Service Centre, further emphasising its focus on customer commitment.

Designed to be as environmentally friendly as possible, the new centre has energy efficient lighting and uses heat generated by the computers to power the air conditioning. Practice Plan Managing Director Steve Turnock explained that the new centre was a big step forward for the company and their dentists. 'The investment in the dedicated Customer Service Centre is only the first phase in providing an even better service and developing stronger links with our practices' he said. 'Over the next six months the whole practice team can look forward to an exciting portfolio of services to assist them in developing their practices, underlining once again our commitment to providing life-long support to all our dentists.'

For more information about Practice Plan, please call 01691677966.

Reader response number 51

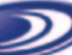

\section{practiceplan}

\section{Bien-Air exclusive}

A two year warranty is being offered by Bien-Air exclusively with the purchase of a twin set of C/A Handpieces - one Blue 1:1 and one Red 1:5 with optics. The set includes a $500 \mathrm{ml}$ can of Spraynet cleaner and a $500 \mathrm{ml}$ can of Lubrifluid lubricant and represents excellent value as well as twice the normal warranty period.

Coupled with the Bien-Air electric micromotor and Optima $T+R$ Digipad, the two contra-angles will cover most dental operations including endodontics. The package is the perfect complement to any existing micromotor installations and of course, the Bien-Air TLC-VLM system offers easy installation of the micromotor/Optima $\mathrm{T}+\mathrm{R}$ for those looking to upgrade. This limited offer is available from June-31 December 2006 and is subject to the handpieces being used with the Bien-Air micromotor. The set is priced at $£ 995$ plus VAT, a saving of $£ 500$ on the recommended retail price. For more information visit www.bienair.com or call Bien-Air on 01306711303. Reader response number 52

\section{Active 10 for good oral health}

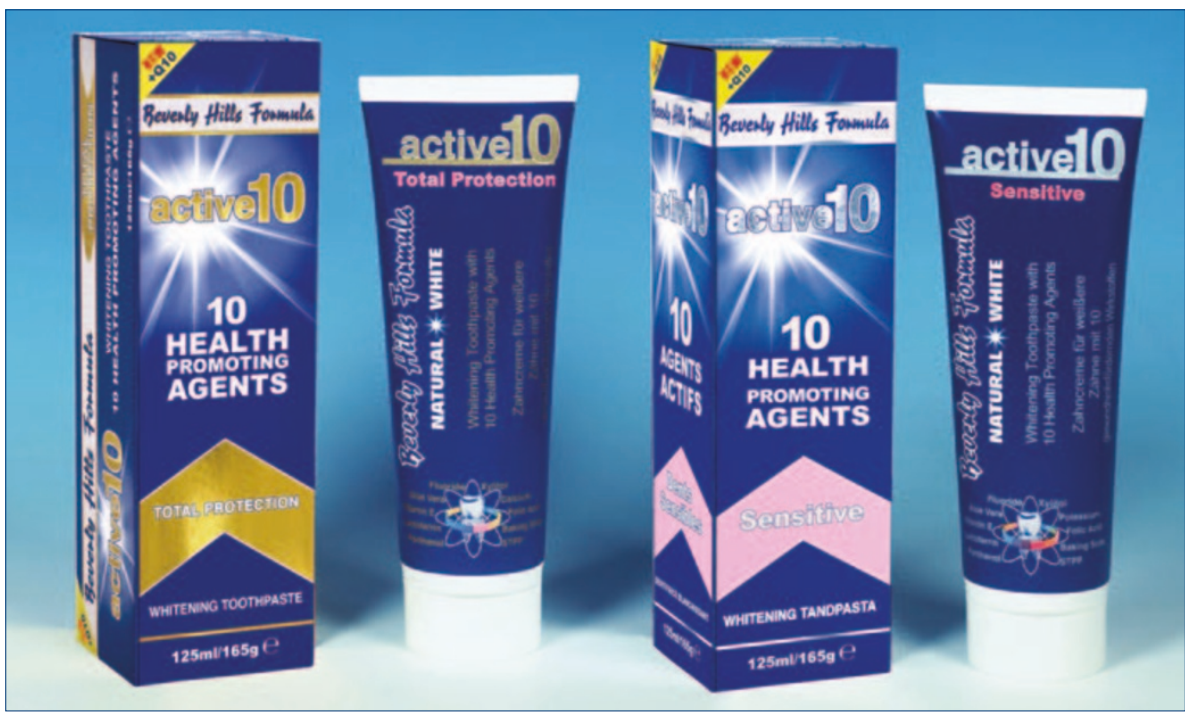

Hills Formula is working with the British Dental Health Foundation to spread the contain ten health procis. improve and maintain a healthy mouth. , the Active10 range contains fluoride, vitamins E and B5, sodium monofluoroen the immune system and aloe vera gel, which contains a tota

Maintaining optimal oral health will not only provide a brighter, healthier smile, but can also increase the odds of living a long and healthy life. For more information visit www.beverlyhillsformula.com or email info@puritylabs.co.uk. Reader response number 50

\section{Physiodens - it's only natural}

VITA Physiodens teeth are a new generation of five-layered acrylic teeth with wear abrasion, aesthetics and fit similar to natural teeth. Now Panadent, together with Dr Stuart Morganstein, has developed the perfect prescription method for VITA Physiodens.

The unique four-dimensional prescription approach uses the patient's facial, profile and plan views as well as the interpupilary distance to create a matrix that allows for fast, easy and precise selection of appropriate teeth in the surgery or laboratory. The method is perfect for complete dentures, partials and long term temporary prostheses including chrome bases. Even in thin section, metal is masked without affecting tooth aesthetics, giving a natural appearance without any cervical 'step' when moving from anterior to posterior teeth. For more information please contact Panadent on 01689881788. Reader response number 53

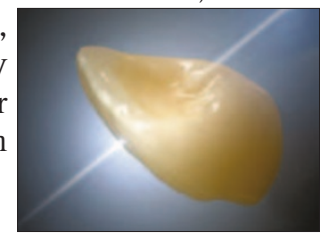




\section{$X-R A Y S$}

\section{1 N

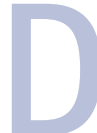 \\ Schick CDR ${ }^{\circledR}$ for a film-free practice}

With the Schick CDR digital X-ray system you can have a totally film-free practice. The new Schick CDRPanX gives remarkably accurate, distortion-free panoramic images thanks to its optimal patient positioning facilities. Patients of any height can be positioned easily, eight image modes help you select the best view and the unique 'Full Vu' mirror reduces patient anxiety and movement.

For intraoral images there is the revolutionary Schick CDR Wireless ${ }^{\mathrm{TM}}$. The World's first cable-free sensor for greater chairside freedom, it uses radio waves to transmit images directly to the computer. The Schick CDR system means low radiation exposure and uses the unique Active Pixel Sensor enhancement to cmos imaging technology, for unsurpassed, razor-sharp image quality.

For further details contact Schick Tech-

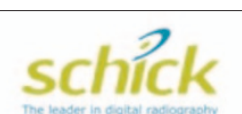
nologies on 01268 733151. number 55

\section{Seeing is believing}

Are you looking for the clearest and most versatile intra-oral camera on the market? Look no further than the Telecam Hawk from DMDS QuickWhite. The Telecam Hawk is the answer to all your needs, allowing you to enhance your treatment plans and enabling complete communication with your patient. The small, portable unit incorporates a lightweight and perfectly balanced single lens handpiece for intraoral access $1 \mathrm{~mm}$ from a tooth to a full smile. Magnified X-ray images can be displayed and there is a built-in single/four 8 frame image capture feature.

The auto iris for light intensity control eliminates reflection and glare, ensuring true colour on all intraoral images. The latest CCD chip and CCU processor with built-in LED illumination produce extremely high resolution and depth of field. Complete with a foot pedal, protection sheaths, light auto on/off holder, video cable, charger, power lead and instruction manual, the Telecam Hawk is made to suit your own personal requirements.

DMDS QuickWhite are currently offering a special promotional offer price of $£ 1,999$ including VAT and a 14" colour TV. For further information please contact DMDS QuickWhite on 01227780009 or visit www. quickwhite.info.

Reader response number 56
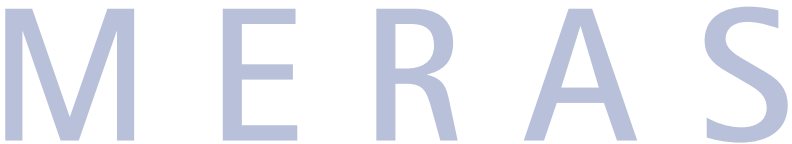

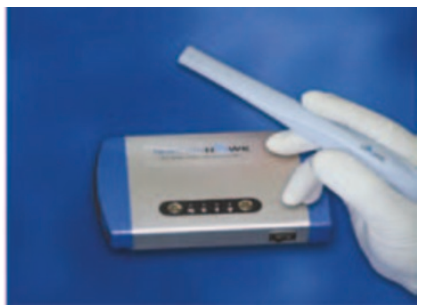

Tailored digital X-ray

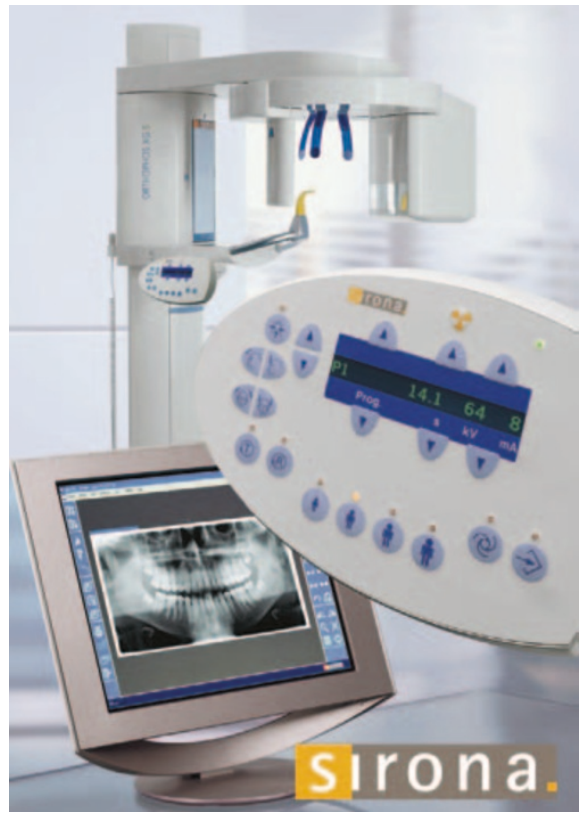

Whether you want cleaner, faster workflow or new sources of earnings potential, Sirona's modular design philosophy for digital X-ray means more flexibility. Not only does digital $\mathrm{X}$-ray eliminate the need for costly consumables, it also streamlines your practice workflow.

Depending on their specific preferences, dentists can opt for a stand-alone configuration or a fully integrated digital practice network. In this case all the digital imaging systems (intraoral and panoramic X-ray and intraoral video) work together in a single seamless system. The stand-alone X-ray unit can be upgraded at any time by adding on extra equipment and has the key advantage that it can be operated either on its own or as an integral part of your practice network.

Each digital module remains fully integrated as your practice expands, not only ensuring a sustained return on investment but also more information on digital X-ray from Sirona, please call 08450715040.

Reader response number 54

\section{Dental Sky for X-rays}

As well as stocking the leading brands of X-ray films and accessories, including Agfa and Kodak, Dental Sky also produce their own label R \& $S$ product line.

Both D and E Speed periapical intraoral films are competitively priced from just $£ 16.45$ for a box of 150 films. Supplied in a soft vinyl packet for patient comfort, these films are suitable for both automatic and manual processing. R \& $\mathrm{S}$ Adhesive Film Mounts are suitable for both child and adult films with 10 pockets for a clearer view. They are supplied in boxes of 25 and are only £19.50 for a box of 250 pockets.

Dental Sky feature X-ray solutions at very cost effective prices and with a compliment of fully trained staff to take your call, you will find dealing with Dental Sky a pleasure. For further information or to request your free full-colour comprehensive catalogue, please contact Dental Sky on 08002944700. Reader response number 57

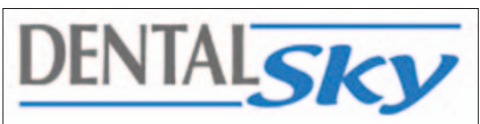




\section{New Progeny JB-70}

The new Progeny JB-70 from Paterson Health Group is part of a new generation of dental imaging that is smarter, more reliable and easier to use. With good looks, simple controls, flawless imaging and a competitive price, the Progeny JB-70 offers the features and performance you need without unnecessary extras.

The unique handle design allows for effortless ergonomic positioning and one hand movement of the tube head, while the smooth gliding arms provide a full range of motion, eliminating unwanted drift. The intuitive anatomical programming ensures instant proficiency for all with a keypad that can be mounted remotely, according to your surgery requirements. The Progeny JB-70 is also compatible with standard film and digital imaging receptors and is designed to provide reliable, razor-sharp X-rays for years to come.

The JB-70 can be installed quickly and easily on almost any wall and is available with a choice of 56", 66" and 76" (total reach) arms. For more information please contact Paterson Health Group on 01594833007 or email sales@paterson.ltd.uk.

Reader response number 58

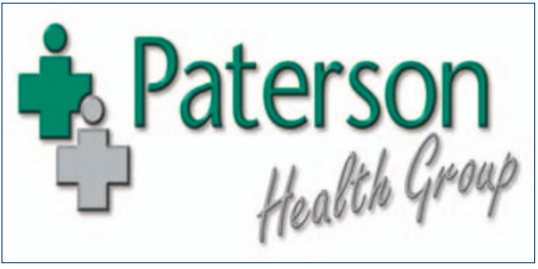

\section{Solutions for X-rays}

Clear radiographs are essential for accurate diagnosis, and Kerr have developed several solutions to enhance your radiography.

The Kerr X-ray Film Holder System saves costly and annoying projection errors. The protection plate prevents the film from bending and therefore significantly reduces distortion of the X-ray. The aiming device, which is closely linked to the film, is moved to the controllable extraoral area, guaranteeing exact reproduction when setting the beam to

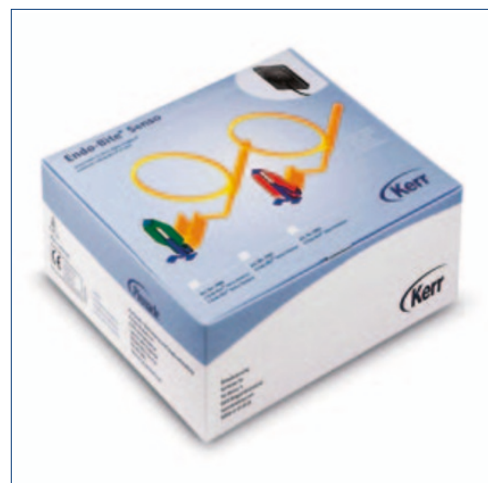
gain consistently successful images.

Kerr manufacture a wide range of X-ray film holders including the Endo-Bite, specifically developed to ensure accurate X-rays with an endodontic instrument in situ, even with a rubber dam in place. The Kerr Super-Bite holds periapical films steady and the Kwik-Bite is ideal for standard bitewings. Kwik-Bite Senseo is also ideal for use with all digital X-ray sensors.

For further information or to place your order, please contact Kerr on 01733892292 or contact your preferred dealer.

Reader response number 59

\section{CR 7400 from KDS}

Kodak Dental Systems has announced the global launch of its new CR 7400 Digital Radiography System, which delivers a full range of digital intraoral and extraoral images based on computer radiography (CR).

The CR 7400 uses standard positioners and techniques and offers a full range of sizes (0-4) for diagnostic examinations. In addition, it easily converts existing film-based panoramic and/or cephalometric systems to digital. Using the same plate sizes as film, the CR 7400 can ensure a fast, easy integration into practices currently using film technology.

Kodak's vast experience in CR medical imaging has allowed the incorporation of many features to improve efficiency and productivity in the dental practice. It is fully integrated with Kodak imaging software for image processing and on-screen display, which can enhance patient education, improve referral communication and streamline practice workflow.

For further information about the new CR 7400 Digital Radiography System or to arrange a demonstration, please call Kodak Dental Systems on 0800895113.

Reader response number 60

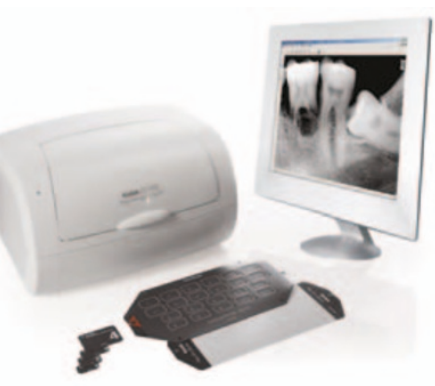

